\title{
Politische Entscheidungszumutungen: Deutsche Einheit 1989/90 und Corona-Politik 2020
}

\author{
Karl-Rudolf Korte
}

Online publiziert: 10. Juni 2020

(C) Der/die Autor(en) 2020

„Diese Pandemie ist eine demokratische Zumutung“ - formulierte Kanzlerin Merkel im April 2020. Dreißig Jahre zuvor kämpfte sich die Politik auch durch Entscheidungszumutungen. Damals war die ostdeutsche Wissenschaftlerin Merkel noch Zuschauerin des Umbruchs. Heute prägt ihr Politikmanagement auch die CoronaPolitik. Die friedliche Revolution 1989 mit der Gestaltung der deutschen Einheit 1990 entwickelte sich als Unikat. Auch die epochale Drastik der Pandemie-Bekämpfung erfolgte ohne historisches Modell für das Politikmanagement. Damals rief man: „Wahnsinn!““. Heute drängt man: „Abstand halten!“. Damals betraf das Krisenmanagement vorrangig die beiden deutschen Staaten. Heute hat sich monothematisch die Pandemie in alle Gesellschaften der Welt gebahnt. Trotz der Unterschiede, finden sich auch Gemeinsamkeiten, wie die besonderen Krisenmomente des Entscheidens verliefen. Um zu verstehen, wie die politische Steuerung eines Stresstests für die Demokratie abläuft, lohnt sich deshalb nochmals der Blick auf das Entscheidungsjahr 1989. Muster, Regeln, Skripte bilden sich aus. Wer die Kontexte von 1989 politikwissenschaftlich einordnet, erkennt die Parallelen zu 2020: Politische Entscheidungen im Lernmodus von disruptivem und turbulentem Wandel unter der Notwendigkeit einer Komplexitäts-Kompetenz der Entscheider (Florack et al. 2021).

„Wir saßen wie Kinder vor dem Weihnachtsbaum und haben uns die Augen gerieben." So umschrieb der damalige Bundesinnenminister Wolfgang Schäuble (CDU)

Univ.-Prof. Dr. K.-R. Korte ( $\square)$

Institut für Politikwissenschaft, Universität Duisburg-Essen, NRW School of Governance,

Lotharstraße 53, 47057 Duisburg, Deutschland 
das Regierungshandeln im Jahr 1989. ${ }^{1}$ Das Bild bezeichnete eine Grundrichtung des Regierungshandelns bis in die ersten Novembertage hinein: Reaktion - keine Aktion; eher zurückhaltendes Krisenmanagement als aktive Unterstützung des Umbruchs in der Deutschen Demokratischen Republik (DDR). Das Entscheidungsjahr 1989 enthielt bereits alle Muster des Politikmanagements in Hochgeschwindigkeitszeiten, die dann 1990 den Beitritt der sogenannten neuen ostdeutschen Bundesländer zum Geltungsbereich des Grundgesetzes möglich machten.

\section{Phase eins: Amtswechsel und Routineverhandlungen}

Mit dem Strom der Ausreisewilligen begann die Talfahrt der DDR - nicht absehbar war mit welchem Ende. Das Kanzleramt konnte 1989 aus den schonungslosen Berichten der Ständigen Vertretung in Ost-Berlin kontinuierlich die Befunde über die innere Verfassung der DDR ableiten. Der steigende Reisedruck war den Berichten und den darin enthaltenen Zahlen über die Antragsteller für Privatreisen zu entnehmen. Die nicht erfüllten ökonomischen Erwartungen der Bevölkerung in der DDR führten zu einer immer größeren politischen Unzufriedenheit, die schließlich - zunächst forciert durch veränderte Gewichtungen im internationalen politischen System - im offenem, friedlichen Massenprotest ihren Ausdruck fand. Der ökonomischen Selbstzerstörung folgte eine Selbstbefreiung.

Die Wirtschaftsmisere, die in den Berichten immer wieder als wichtige Quelle der Unruhe genannt wird, führte nicht unmittelbar zum Zusammenbruch des gesamten Systems, aber die politisch wie ökonomisch bedingte Akzeptanzkrise war ein wichtiger Katalysator des Umbruchs. Mehrdimensional schien das politische System der DDR herausgefordert, wie das Kanzleramt einem Bericht der Ständigen Vertretung entnehmen konnte.

Die Flüchtlingswelle war der eigentliche Vorbote des Umbruchs. Doch sie wurde von der Bundesregierung nicht als solcher wahrgenommen. Die Zahl der Ausreisewilligen belastete im Frühjahr 1989 das Verhältnis der beiden deutschen Staaten zueinander. Doch noch galt es, die Politik der behutsamen, pragmatischen kleinen Schritte für menschliche Erleichterungen und zur Linderung der Teilung fortzusetzen. Aus einer nüchtern-pragmatischen Lagebeurteilung heraus folgte die Deutschlandpolitik dem gewohnten Regierungshandeln: mühsame Normalisierung, Proteste bei Menschenrechtsverletzungen, Beibehaltung und Betonung der Grundpositionen. Augenmaß und Umsicht waren gefordert, ohne Drohungen oder Warnungen. Schäuble, später Rudolf Seiters (CDU) als nachfolgender Kanzleramtsminister, traf weiterhin unregelmäßig den Staatssekretär des Ministeriums für Außenhandel der DDR, Alexander Schalck. Dabei besprach man häufig und relativ offen die Finanzprobleme der DDR. ${ }^{2}$

\footnotetext{
1 Vgl. Karl-Rudolf, Korte, Deutschlandpolitik in Helmut Kohls Kanzlerschaft. Regierungsstil und Entscheidungen 1982-1989, Stuttgart (1998, S. 438). Nachfolgende Details mit Bezug zu den Jahren 1989/90 finden sich alle in diesem Buch, das auch mit der Auswertung der Akten des Kanzleramtes eine authentische Rekonstruktion des Politikmanagements des Kanzleramtes im Entscheidungsjahres enthält.

$2 \mathrm{Zu}$ den politischen und ereignishaften Details der deutschen Einheit vgl. Karl-Rudolf Korte (1994): Die Chance genutzt. Die Politik zur Einheit Deutschlands, Frankfurt/New York 1994 sowie die Ge-
} 
Seiters' Augenmerk galt nach der Amtsübernahme nicht vorrangig dem innerdeutschen Beziehungsgeflecht. Er wollte institutionell und inhaltlich Kurs halten. Während Schäuble den Typ des kühl und politisch-strategisch handelnden Managers verkörperte, repräsentierte Seiters eher den Hintergrundtyp, der unauffällig, unpolemisch und auch formeller im Umgangston seine Kreise zog. Der Bundeskanzler hatte Seiters nicht ins Amt berufen, um deutschlandpolitische Innovationen voranzutreiben. Kohl wollte einen ebenso wie Schäuble äußerst loyalen wie effektiven Organisator und Koordinator, der des Kanzlers informellen Regierungsstil personifizierte. Kohl regierte deutschlandpolitisch über den Kanzleramtsminister. Daran sollte sich nichts ändern.

Bezüglich der deutschen Frage erhielt Seiters die Devise: Weiter am Stabilitätskurs festhalten. Deutschlandpolitik sollte bis auf Widerruf ein Reparaturunternehmen an der ansonsten akzeptierten Zweistaatlichkeit bleiben. Dem Kanzleramtsminister konnte die angedeutete Alternative nicht empfohlen werden, denn wer garantierte zum damaligen Zeitpunkt, dass eine ängstlich-defensiv an der Macht hängende SED-Führung auf Bonner Druck nicht mit weiterer Verhärtung im Innern reagiert hätte?

\section{Phase zwei: Krisenmanagement - Massenausreise und Botschaftsflüchtlinge}

Die zweite Phase des Regierungshandelns ist angesichts der Besetzung der Ständigen Vertretung sowie einiger deutscher Botschaften durch ausreisewillige DDRBürger durch Krisenmanagement gekennzeichnet. Die in den westdeutschen Vertretungen versammelten Menschen nannte man amtlich „Botschaftsflüchtlinge“. Die beschleunigten Abläufe erforderten Krisenbewältigung durch informelle kleine Zirkel und in zahllosen Einzelgesprächen, die der Kanzler führte oder führen ließ. Doch auf das Tempo drückte der Kanzler in dieser Phase keineswegs.

Die Ausreiseproblematik und die sich daran anschließende Massenflucht aus der DDR stellte weniger eine deutschlandpolitische als primär eine innenpolitisch zu bewältigende Herausforderung der Regierung Kohl dar. Ab dem 2. August 1989 erfolgte im Kabinett wöchentlich eine informationspolitische Lageeinschätzung über die praktischen Belange der Ausreiseproblematik. Die Diplomatie erfolgte als innenpolitisch motivierte Krisenbewältigung mit deutschlandpolitischen Folgerungen, die jedoch niemand zum damaligen Zeitpunkt konkret im Sinne einer Wiedervereinigungsoption problematisierte. Die Bonner Politik äußerte tiefe Besorgnis angesichts der Dramatik des Massenexodus' und rief gleichzeitig zur Zurückhaltung auf. Der Bundeskanzler reagierte zögerlich.

Die Botschaftsbesetzungen waren überraschend gekommen. Ihre Größenordnung überstieg bei weitem die früheren Zwischenfälle. Auch deshalb erwiesen sich die Gespräche des Kanzleramtes mit der ostdeutschen Führung über eine einvernehmliche Lösung als äußerst schwierig. Unmissverständlich drängte Kohl auf eine Lösung,

samtdarstellungen in: Werner Weidenfeld/Karl-Rudolf Korte (Hrsg.): Handbuch zur deutschen Einheit 1949-1989-1999, 4. Auflage, Campus: Frankfurt a.M./New York (1999). 
wobei er erstmals auch die Motive der Ausreisewilligen offen thematisierte. Das musste Honecker als grobe Einmischung in die inneren Angelegenheiten auffassen, aus denen sich Bonn bisher diplomatisch stets zurückhielt. Honecker pochte auf die Souveränität der DDR und wiederholte seine bekannten Grundsätze bezüglich der Nichtzuständigkeit Bonns für die Bürger der DDR. Dennoch stimmte er einem vertraulichen Gesprächskanal in dieser Sache zu.

Öffentlich erklärte der Bundeskanzler am 22. August 1989, dass er zu einem Treffen mit Honecker bereit sei, wenn damit eine Erleichterung für die Menschen in der DDR erreicht werden könne. Gleichzeitig drohte ein neuer Krisenherd zu entstehen. Die westdeutschen Botschaften in Prag, Budapest und Warschau füllten sich ebenfalls mit Flüchtlingen aus der DDR. Auch deshalb wollte die DDR bei der Lösung der Besetzung der Ständigen Vertretung in Ost-Berlin kompromisslos bleiben. Die anzustrebende Lösung musste Vorbildfunktion für die anderen Botschaften haben.

Erst am 31. August sicherte die DDR schließlich den Botschaftsflüchtlingen zu, dass sie nach Verlassen der deutschen Vertretung einen Ausreiseantrag mit Rechtshilfe stellen könnten. Doch die Mehrzahl der Ausreisewilligen ließ sich von dem Vorschlag nicht überzeugen. Eine wirksame Verteidigungsstrategie war das nicht für die DDR, wie der weitere Zulauf in den Ostblock-Botschaften belegte.

Bundeskanzler Kohl plädierte in der Kabinettsitzung am 24. August nachhaltig erneut für öffentliche Zurückhaltung, da er eine Sogwirkung auf alle deutschen Botschaften im osteuropäischen Raum befürchtete. Er erklärte, dass sich vor diesem Hintergrund eine Politik der Destabilisierung verbiete. Unverrückbar und von der jeweiligen Situation unbeeinflusst sollte die vorrangige Aufgabe der Politik allerdings bestehen bleiben, allen Deutschen das Recht auf Zuflucht und Unterstützung zu gewähren. Was Bundeskanzler Helmut Kohl und Außenminister Hans-Dietrich Genscher (FDP) wussten, aber noch nicht öffentlich verwenden konnten: Ungarn war bereit, bis Mitte September 1989 die Grenze zu öffnen. Die ungarische Regierung wollte von da an keinen DDR-Bürger gegen seinen Willen aus Ungarn in die DDR zurückschicken. Bei einem Geheimtreffen auf Schloss Gymnich bei Bonn hatten dies Ministerpräsident Miklos Nemeth und sein Außenminister Gyula Horn vertraulich im Gespräch mit Kohl und Genscher zugesichert. Der konkrete Termin stand noch nicht fest. Außerdem zweifelte Kohl, ob der ungarische Ministerpräsident dieses Vorhaben im Warschauer Pakt durchsetzen könnte. Denn seine Mitglieder hatten sich verpflichtet, Flüchtlinge auszuliefern. Nach einem Telefonat mit Gorbatschow schien Kohl beruhigt zu sein. Moskau stand auf der Seite der Ungarn.

Neues Krisenmanagement in der deutschen Botschaft in Prag war unterdessen erforderlich geworden. Dort stieg die Zahl der Ausreisewilligen Ende September 1989 auf mehrere Tausend an. Die Botschaftsbesetzungen hatten damit auch eine nachhaltige internationale Wirkung. Die DDR wiederum wollte weitere Ansehensverluste vermeiden, weil die geplanten Feierlichkeiten zum vierzigjährigen Bestehen der DDR unmittelbar bevorstanden.

Noch am Nachmittag des 30. September flogen Genscher und Seiters nach Prag. Bis zu ihrem Eintreffen sollte, wie im Gespräch mit Horst Neubauer (DDR-Diplomat) vereinbart, strengstes Stillschweigen gewahrt werden. Kohl hinderten gesundheitliche Probleme daran, selber nach Prag zu fliegen. Genscher nutzte die 
Chance, um sich weltweit politisch in Szene zu setzen. Auf dem Balkon der Botschaft verkündete er die Erfolgsmeldung über die bevorstehende Ausreise, die auch Fernsehgeschichte machte. Trotz vereinbarter Diskretion ließ Genscher bereits während des Fluges über die Presseagenturen melden, dass er auf dem Weg nach Prag sei. Der Anteil Genschers und Seiters' am Verhandlungsdurchbruch war gleich groß, allerdings erntete nur der Außenminister die Lorbeeren des Erfolgs. Kohl zog daraus seine Konsequenzen für das weitere bilaterale Krisenmanagement mit Genscher. Um nochmaliges Vorpreschen des Außenministers zu verhindern, weihte Kohl ihn später nicht in das Zehn-Punkte-Programm ein. Am Morgen des folgenden Tages verließ der erste von insgesamt sechs Sonderzügen Prag in Richtung Hof. Die DDR hatte politisch kapituliert. Sie musste dem Ausreisedruck immer mehr nachgeben. Die Bevölkerung interpretierte jede Konzession als die letzte Gelegenheit zu entkommen, denn unklar blieb, wie sich die politische Lage, vor allem aber auch das Verhalten Moskaus, entwickeln würde.

Der Umbruch in der DDR war durch die Massenflucht mit ausgelöst worden. Eine Kettenreaktion aus Flucht und Protest schien nicht mehr aufzuhalten. Großdemonstrationen in Ost-Berlin, Leipzig und Dresden folgten unmittelbar. Berichte, die das Kanzleramt aus der Sowjetunion erhielt, ließen darauf schließen, dass Moskau die Ausreisewelle beobachtete und offiziell verurteilte, ohne sich jedoch einzumischen. Die Unterstützung für die DDR seitens der Sowjetunion wurde zu diesem Zeitpunkt als äußerst schwach eingeschätzt.

In Bonn standen alle Zeichen auf Krisenbewältigung. Vorrangig sollte den Ausreisewilligen geholfen werden. Doch gegenüber der SED-Führung hielt man sich noch deutlich mit Kritik und weiteren Forderungen zurück. Der Zeitpunkt für eine Wende der Deutschlandpolitik im Sinne einer operativen Wiedervereinigungspolitik war noch lange nicht gekommen. Der Mauerfall erschien vollkommen unrealistisch. Beim Krisenmanagement dieser Zeit informierte Seiters auch den Parteivorstand der SPD, wie er es im Kabinett am 4. Oktober allen Kollegen mitteilte. Kohl und Genscher appellierten an gleicher Stelle, nach ausführlichem Bericht von Seiters über die Lage in den Botschaften, auf alles zu verzichten, was die ohnehin bis zum Äußersten angespannte Situation in der DDR weiter verschärfen würde. Nach Möglichkeit sollten in der DDR die beruhigenden Elemente gestützt werden.

\section{Phase drei: Einmischung in die inneren Angelegenheiten}

Massenflucht nach der Grenzöffnung Ungarns am 11. September und einsetzende Massenproteste in der DDR waren die Ouvertüre für den Fall der Mauer. Aus DDRSicht wurde das Thema der politischen Reformen zu einer existentiellen Bedrohung. Die Staatskrise spitzte sich im Umfeld des 40jährigen Staatsjubiläums zu. Dass der Machtverlust auch gleichzeitig zum Staatsverlust führen sollte, ahnten nur wenige. Die aus Anlass der Jubelfeier inszenierte Öffentlichkeit des Regimes steigerte noch einmal den Protest. Die Feier wurde zum Offenbarungseid: Das Volk auf der Straße rief „Gorbi, hilf uns“ - gegen die eigene Führung.

Aber erst ab Anfang November lässt sich für den Bundeskanzler ein konzeptioneller Wandel in der Deutschlandpolitik feststellen: Der diplomatischen Zurückhaltung 
folgte das Drängen auf politische Veränderungen, für die Devisenzahlungen zur ökonomischen Stabilisierung in Aussicht gestellt wurden. Die Gründe für diesen qualitativen Wandel hingen mit der dramatischen Zuspitzung der inneren Verhältnisse in der DDR zusammen.

Noch stand Kohl den Ereignissen in der DDR als Zuschauer gegenüber. Die Lage schien zum damaligen Zeitpunkt unkalkulierbar. So kam es am Abend des 17. Oktober überraschend zu Honeckers Absetzung als Generalsekretär des Zentralkomitees der SED durch einstimmiges Votum des Politbüros. Sein Nachfolger wurde Egon Krenz, der außerdem am 24. Oktober 1989 von der Volkskammer zum Vorsitzenden des Staatsrates und des Nationalen Verteidigungsrates gewählt wurde. In seiner ersten Rede als Generalsekretär sprach Krenz von der „Wende“, die er einleiten wollte, um die ,politische und ökonomische Initiative“ wiederzuerlangen.

Der Bundeskanzler nahm die Wahl von Krenz mit vorsichtiger Hoffnung auf. Bei allen politischen Akteuren war eine gewisse Erleichterung zu spüren. Diese Gefühle entsprangen allerdings eher dem Wunsch nach Veränderungen als der faktischen Lage. Kohl telefonierte schließlich am 26. Oktober 1989 mit Krenz. Beide vereinbarten, sich wechselseitig telefonisch zu informieren, wenn es die Lage erfordere. Außerdem sollte der Sonderkanal erhalten bleiben. Kohl versicherte Krenz zunächst, dass die Bundesregierung an einer Destabilisierung der DDR nicht interessiert sei. Konkret stellte der Bundeskanzler folgende Forderungen: Neuregelung der Reisefreiheit; Amnestie für die bei den Demonstrationen festgenommenen Bürger der DDR sowie für diejenigen, die wegen des illegalen Grenzübertritts zur Republikflucht verurteilt wurden; Bereitschaft zur positiven Lösung für die sogenannten Botschaftsflüchtlinge.

Als Geschäftsgrundlage schlug der Bundeskanzler vor, diese prinzipiellen Unterschiede nicht zu thematisieren, sondern zunächst die praktische und pragmatische Kooperation zur Zusammenarbeit zu pflegen. Kurz nach dem Gespräch telefonierte Kohl mit Gorbatschow und entnahm dessen Worten, dass Krenz keinerlei Rückhalt in Moskau besaß.

Krenz war kein Reformator. Dennoch genehmigte er die Großdemonstration auf dem Berliner Alexanderplatz am 4. November. Im Mittelpunkt der Reden standen die Reform des Systems und der Ausbau der politischen Selbstbestimmung. Bundeskanzler Kohl nahm dies zum Anlass, um seiner Deutschlandpolitik eine neue Qualität zu geben, was am 6. November 1989 im Bonner Gespräch zwischen Seiters, Schäuble und Schalck unter Beweis gestellt werden sollte. Schalck forderte angesichts der ruinösen DDR-Wirtschaft Milliardenbeträge von der Bundesrepublik. Gleichzeitig signalisierte er Bereitschaft, dafür auch einen politischen, nicht nur humanitären Preis zu zahlen. So deutete er substantielle politische Reformen an, unter anderem auch die Möglichkeit freier Wahlen. Schalck gab zu erkennen, dass er auf eine Botschaft des Bundeskanzlers warte. Es würde sich der Bericht zur Lage der Nation anbieten, der für den 8. November 1989 auf der Tagesordnung des Bundestages stand.

Im Kanzlerbungalow entschieden sich Kohl, Seiters und Schäuble schließlich am Abend des 6. November die bisherige Zurückhaltung aufzugeben. Für die bereits fertig vorliegende Rede zum Bericht zur Lage der Nation im geteilten Deutschland, die Kanzler Kohl am 8. November im Bundestag halten wollte, formulierten 
sie abends folgenden Passus: „Ich erkläre gegenüber der neuen SED-Führung meine Bereitschaft, einen Weg des Wandels zu stützen, wenn sie zu Reformen bereit ist. Kosmetische Korrekturen genügen nicht (...). “3 Die neue Qualität bestand im erweiterten Hilfsangebot bei einschneidenden Reformen.

Operativ wollte Kohl jedoch zunächst nichts ändern. Er warnte vor übereilten Wiedervereinigungserwartungen; die Reform des politischen Systems, nicht die Einheit, machte er zur Bedingung für finanzielle Hilfeleistungen. Am 9. November wurde die Berliner Mauer geöffnet. Günter Schabowskis Presseerklärung zum Reisegesetz und zur Tagung des Zentralkomitees der SED interpretierten Tausende noch in derselben Nacht als Öffnung der Grenze. Sie behielten recht.

\section{Kohls Entscheidungsverhalten}

Die Dynamik des Gesamtprozesses 1989 war weder vorhersehbar noch von Bonn aus steuerbar. Die Bürger der DDR selbst avancierten zu den Akteuren des Umbruchs. In dem Maße, wie sich die Situation dramatisch zuspitzte, waren Kohls persönliche Entscheidungen mehr denn je und in schneller Abfolge gefordert. Doch das Einflussnehmen und das Richtunggeben erfolgten zunächst äußerst zögerlich, wie gezeigt werden konnte. Kohl vermied alles, was das Tempo des unkalkulierbaren Umbruchs gesteigert hätte. Als er schließlich eine qualitative Veränderung seiner Politik gegenüber der DDR ankündigte, geschah dies aus einer Position wiedergewonnener innerparteilicher Stärke heraus. Auch die erste Reise ins demokratische Polen, die Kohl am 9. November antrat, konnte er angesichts der sensiblen Konfliktlage innerhalb der Union zur polnischen Westgrenze nur auf dem relativ stabilen Fundament eines innerparteilichen Rückhalts durchführen. Doch die Führungsstärke, die der Bundeskanzler im November 1989 unter Beweis stellte, täuschte darüber hinweg, dass Kohl über viele Monate des Jahres 1989 zunächst das machtpolitische Feld zurückerobern musste, um neuen Handlungsspielraum zu erlangen. Die Dramatik der Ausreisewelle und des Umbruchs in der DDR erlebte der Kanzler in einer persönlich, wie auch innerparteilich höchst angeschlagenen Verfassung. Ränkeschmiede vereinten sich im Versuch, Kohl als Vorsitzenden auszubooten.

Kohl war im doppelten Sinne herausgefordert: Deutschlandpolitisch durch die Ereignisse in der DDR und parteipolitisch durch heftige Kritik am konturlosen Kurs des Parteivorsitzenden. Die Machtfrage für Kohls Kanzlerschaft stellte sich in einer bis dahin nie dagewesenen Schärfe. Seine Herrschaft war weder dauerhaft abgesichert noch präsidial überhöht. Die Herausforderung hatte vielschichtige Hintergründe:

So sah sich Kohl im Sommer 1989 in nahezu aussichtsloser Position und auf dem persönlichen Tiefpunkt seiner Karriere angelangt. Seit Frühjahr 1989 spielte er ernsthaft mit dem Gedanken, seine Kanzlerschaft zu beenden. Kohl taktierte schließlich bis zu seinem Sommerurlaub (21.07. bis 18.08.1989) am Wolfgangsee parteipolitisch nur aus der Defensive heraus. Seine Machtposition stärkte er während dieser Phase, indem er von dort aus, mittels intensiver Telefondiplomatie, wichtige Entscheidungen vorbereitete. Von außen betrachtet, saß er dort wieder die Proble-

\footnotetext{
3 Bulletin der Bundesregierung vom 09.11.1989, S. 723-739.
} 
me aus, wie der allgemeine Vorwurf lautete. In Wirklichkeit agierte er zwar extrem vorsichtig, aber keineswegs stoisch langsam. Neue, sich ständig ändernde Lageinterpretationen erhielt Kohl über sein feingefiltertes persönliches Frühwarnsystem aus allen Flügeln seiner Partei. Manch Bonner Journalist zeigte bereitwillig doppelte Loyalitäten, um mal für Kohl, mal für Genscher instrumentalisierbar zu sein. Dieses gesamte Kommunikationssystem bescherte permanente Informationseingänge, die wendige Gegenreaktionen erforderten und zum Terminus des „Aussitzens“ nicht passten. Erst als die Ergebnisse der Sondierungen für Kohl am Wolfgangsee 1989 feststanden, der parteipolitische Widerstand kalkulierbar schien, Positionen wichtiger Vertrauter eingeholt und Kompromissmöglichkeiten eingeschätzt waren, wagte er sich aus der Deckung und ging mit seinen Alleinentscheidungen in die Offensive. Das betraf sowohl parteipolitische als auch deutschlandpolitische Überlegungen.

Den bevorstehenden Bundestagswahlkampf entschied Kohl zum Deutschlandthema zu führen. Er erkannte in dieser Mischung die Chance zum weiteren Machterhalt. Es musste ein für die Partei einigendes und zukunftsweisendes Thema zugleich sein. Nur so war zu erklären, dass Kohl nach seiner Rückkehr aus dem Urlaub geradezu kämpferische Töne zum Deutschlandthema anschlug. Schwieriger gestaltete sich der wahltaktische Hintergrund. Konnte man mit dem Deutschlandthema Mehrheiten gewinnen? Denn im Sommer 1989 kippte zunächst die Stimmungslage in Westdeutschland: Aus der überschäumenden Sympathie mit den DDR-Übersiedlern und auch den deutschstämmigen Aussiedlern aus Osteuropa entstand allmählich eine kritische Distanz.

Wie sehr die partei- und wahltaktische sowie die deutschlandpolitische Entwicklungslinien zusammenliefen, zeigte der Bremer Parteitag (11. bis 13.09.1989) der CDU. Am Abend vor Beginn des Parteitages, beim traditionellen Presseempfang, konnte Kohl mitteilen, dass er soeben vom ungarischen Ministerpräsidenten erfahren hatte, dass ab null Uhr des gleichen Tages Deutsche aus der DDR von Ungarn aus in ein Land ihrer Wahl ausreisen können. Der Bundeskanzler hatte bereits wenige Tage zuvor davon gehört, dass der erste Ausreisetag unmittelbar bevorstehen sollte; denn der ungarische Botschafter Horvath hatte am 6. September 1989 Frank Elbe, den Mitarbeiter Genschers, davon in Kenntnis gesetzt, dass bis zum 10. September eine entsprechende Regelung gefunden werden sollte. Genscher informierte daraufhin telefonisch Kohl, Wolfgang Mischnick (Fraktionsvorsitzender FDP) und den SPDParteivorsitzenden Hans-Jochen Vogel. Kohl erwähnte in seinem Erinnerungsbuch den SPD-Abgeordneten Karsten Voigt, dem er dieses Datum verdankte. Denn Voigt hatte offenbar vertrauliche Informationen an die Presse in Ungarn weitergegeben, die wiederum Nemeth und Horn dazu veranlassten, den Termin kurzfristig zu verschieben. Außerdem wurde in den Nachrichten davon berichtet, dass sich die Ausreise deshalb verzögere, weil Ungarn mehr Geld von Deutschland wollte.

Erfolgsgestärkt auch durch die Ausreisemeldung setzte sich Kohl am anderen Tag mit seinem Vorschlag, Volker Rühe zum neuen Generalsekretär zu machen, überzeugend durch, obwohl er selbst bei der Wahl des Parteivorsitzenden das schlechteste Ergebnis seiner Amtszeit seit 1973 hinnehmen musste. Seine Widersacher Lothar Späth und Heiner Geißler aber straften die Delegierten mit Stimmenverweigerung. Zweimal griff Kohl während seiner Parteitagsrede kämpferisch das Deutschlandthema auf. Dabei vermittelte er eine emotionale Botschaft, keine konkrete Wie- 
dervereinigungsrhetorik, doch ein Gespür für das packende Thema der nächsten Bundestagswahl: Offenbleiben sowohl für mögliche Änderungen im Ost-West-Gefüge als auch für eine Betroffenheit gegenüber den menschlichen Schicksalen, mit denen die Übersiedler zunehmend die etablierten und saturierten Westdeutschen konfrontierten. Der Bundeskanzler hatte seit seiner Wiederwahl zum Parteivorsitzender Handlungsmacht zurückgewonnen und Führungsstärke bewiesen; seine Widersacher Geißler und Späth wurden abgestraft; Stoltenberg hatte im neuen Ressort weniger Einfluss als vorher. Koalitionspolitisch hatte sich auch die Abberufung Zimmermanns beruhigend ausgewirkt. Die CSU war durch Waigel besonders dezidiert eingebunden.

Bundeskanzler Kohl ordnete mit dem Parteitag Mitte September sein Haus neu, besetzte das Deutschlandthema als Wahlthema und stellte Führungsstärke unter Beweis. Für das Regierungshandeln war er auf das unionsinterne Machtpolster angewiesen. Er konnte sich fortan stärker auf das Krisenmanagement, das durch die Massenflucht aus der DDR notwendig wurde, konzentrieren. Er besaß den Kompass und ganz offensichtlich auch die notwendige Komplexitäts-Kompetenz, um sich den rasenden Herausforderungen in den folgenden Monaten bis zur ersten gesamtdeutschen Bundestagswahl vom 2. Dezember $1990 \mathrm{zu}$ stellen. Aus seiner Sicht war der Etappensieg bei den ersten freien Wahlen in der DDR (Volkskammerwahl vom 18.03.1990) die Grundlage für den Gewinn der Bundestagswahlen (Weidenfeld und Korte 1991). Es stärkte vor allem seine Verhandlungsposition für das außerordentliche Politikmanagement im Zeitraffer der kommenden Monate: Am 23. August 1990 beschloss die Volkskammer den Beitritt der DDR zur Bundesrepublik. Der sogenannte Einigungsvertrag wurde am 20. September 1990 im Bundestag verabschiedet. Die Einigung mit den Alliierten Siegermächten des Zweiten Weltkrieges konnte im Rahmen von „Zwei plus vier“ Verhandlungen am 19. September unterzeichnet werden. Der Beitritt der DDR erfolgte am 3. Oktober 1990.

\section{Kohls Regierungshandeln}

Das deutschlandpolitische Regierungshandeln in den Entscheidungsjahren 1989/90 stand normativ und operativ in der Kontinuität der bisherigen Politik. Nüchtern, pragmatisch und von einem breiten Konsens getragen, schien es bis zum Sommer 1989 ausschließlich um Reparaturarbeiten an der ansonsten akzeptierten Zweistaatlichkeit zu gehen. Routineverhandlungen liefen ebenso zu den traditionellen Themen der deutschlandpolitischen Agenda, wie auch der Tonfall im deklaratorisch-symbolischen Bereich keine Änderungen anzeigte. Das Netzwerk an Verträgen, Abkommen und Absprachen entsprach den Grundlinien der Politik, sich auf das Machbare im Sinne von menschlichen Erleichterungen und der Regelung praktischer Probleme zu konzentrieren. Der Balanceakt der Offenhaltung der deutschen Frage bei gleichzeitiger Kommunikations- und Interessenverflechtung mit der DDR schien aufrecht erhaltbar.

Mit den Botschaftsflüchtlingen und der Massenausreise von DDR-Bürgern setzte für das Regierungshandeln eine neue Phase ein. Die vom Kanzler, Kanzleramtsminister und Außenminister gesteuerte Krisendiplomatie, die in kleinen informellen 
Entscheidungskreisen abgestimmt wurde, setzte ab Sommer 1989 neue Akzente. Die Telefondiplomatie und unzählige informelle Einzelgespräche sicherten den Kurs auch international ab. Der Kanzler war persönlich gefordert.

Kohl rief zur Zurückhaltung auf, betrachtete äußerst vorsichtig die politischen Entwicklungen in der DDR und unterließ es, den Druck auf die SED-Führung zu verstärken. An den deutschlandpolitischen Grundpositionen nahm er keine Änderungen vor. Noch schien dem Kanzler jeder Druck auf die DDR zu einer Verhärtung im Innern der DDR zu führen. Ein solcher Kurs hätte seiner Meinung nach das bisher Erreichte eher gefährdet als gefördert.

Auf dem Höhepunkt der Massenproteste in der DDR - Anfang November als die Massenausreise längst eine internationale Dimension angenommen hatte - entschied sich Kohl nach telefonischer Rückversicherung mit Gorbatschow und Bush für eine qualitative Änderung seiner bisherigen Deutschlandpolitik. Er mischte sich offen in die inneren Angelegenheiten der DDR ein und forderte sichtbare politische Reformen. Er setzte dazu die wirtschaftliche Stärke der Bundesrepublik ein, um die DDR zu verändern: Umfassende finanzielle Hilfe koppelte er an das Ende des Machtmonopols der SED. Über diese qualitative Änderung entschied Kohl in Abstimmung mit Seiters und Schäuble informell an einem der abendlichen Treffen im Kanzlerbungalow. Es bedeutete erstmals den Versuch, aus der Rolle des Zuschauers zum Akteur des Prozesses zu werden.

Kohls extrem vorsichtiger Kurs änderte sich sukzessive auch in dem Maße, in dem er innerparteilich seine Macht wieder gestärkt hatte und als Wahlkämpfer in den eigenen Reihen integrierend mit dem Deutschlandthema wirken konnte. Auf dem Tiefpunkt seines persönlichen Ansehens und angesichts innerparteilicher Putschpläne erlebte er das Entscheidungsjahr aus der Defensive heraus. Das zögerliche Regierungshandeln mit den zahlreichen Beruhigungsformeln gegenüber den Ausreisewilligen hing auch mit dieser innerparteilichen Schwächeperiode des Parteivorsitzenden Kohl zusammen. Erst als er die notwendigen parteipolitischen Entscheidungen in seinem Sinne vorbereitet hatte, startete er in die deutschlandpolitische Offensive. Getrübt wurde dieses Handeln durch den koalitionspolitischen Zwist über die Anerkennung der polnischen Westgrenze. Doch mit dem Bericht zur Lage der Nation im geteilten Deutschland am Tag vor der Maueröffnung versöhnte er sowohl die deutschlandpolitischen wie auch die ostpolitischen Fronten.

Deutschlandpolitisches Regierungshandeln im Entscheidungsjahr zeigte die Abhängigkeiten und Zwänge, denen sich der Kanzler auf Grund parteipolitischer und koalitionspolitischer Herausforderungen ausgesetzt sah. Die Komponenten des ,Systems Kohl" blieben trotz der nationalen wie auch internationalen Herausforderung unverändert. Der Regierungsstil blieb der Kontinuität des informellen, stillen und zuallererst machtabgesicherten Regierungshandelns treu und zeigte damit die extreme konsensdemokratische Abhängigkeit des Kanzlers von seiner parteipolitischen Machtbasis. Ohne das für Kohl typische informelle Vorarrangement und sein dichtes Kommunikationsnetz als Frühwarnsystem wäre er vermutlich als Parteivorsitzender 1989 gescheitert. Seine Gegner aber antizipierten sowohl Kohls unterstellte Machtposition als auch seine Überlegenheit und nahmen damit auch teilweise bereits das Scheitern ihres Putsches vorweg. Ohne den Parteivorsitz wäre Kohl ein Kanzler auf Abruf gewesen, ohne Macht und Einfluss, ein Spielball der Koalition. Als Krisenlot- 
se verschaffte er sich in der historischen Konstellation besondere Anerkennung. Die Muster seines machtgestützten Politikmanagements blieben zwar gleich, aber sowohl das Sujet als die Intensität der Krisenentscheidungen waren beispiellos. Die Bewährungsprobe der deutschen Demokratie hing mit Widerstands- und Lernfähigkeiten zusammen, die verstärkend wirkten. So liegen - bei allen berechtigten Einwänden gegenüber historische Vergleichen, durchaus mit abstrakten Herangehen Parallelen vor, wenn man sich 1989/90 und 2020 skizzenhaft ansieht.

\section{Analoge Entscheidungszumutungen nach 30 Jahren}

Das strukturelle Entscheiden unter den Bedingungen von Unsicherheit und Risiko verbindet beide Zeitausschnitte. Der Anlass, die Vehemenz und die Tiefe des Nicht-Wissens variieren, nicht jedoch die Notwendigkeit von Entscheidungszumutungen für die Spitzenpolitiker (Korte 2020). Die damalige und heutige formative Phase des politischen Entscheidens stehen unter dem permanenten Druck wachsender Komplexität, zunehmender Unsicherheit, steigendem Nichtwissen, dynamischen Zeitbeschleunigungen und exponentiellen Risikoerwartungen. Das deutsche politische System hat mit robuster Funktionsfähigkeit einer besonderen Gouvernementalität darauf professionell reagiert. Als Resilienzstärke erweist sich einmal mehr die Kombination aus Politikmanagement und Verwaltungsaufbau: „Die Dualität von struktureller Fragmentierung im Organisatorischen und Vereinheitlichung über Rechtsetzung und weitere Mechanismen der Reintegration ist ihr wesentlicher Kern“ (Seibel 2017, S. 173). Träger dieses deutschen Sonderwegs sind vor allem die drei gebietskörperschaftlichen Ebenen von Bund, Ländern und Gemeinden. Die robuste Funktionsfähigkeit dieses verwaltungstechnischen Politikmanagements führte auch vor 30 Jahren zu einer intensiven Koordination zwischen den Ministerpräsidenten und dem Kanzler. Die Bundesländer verbündeten sich zusätzlich über Patenschaften mit den fünf neuen Ländern im Osten.

Abstrakt zeigen sich im Entscheidungshandeln 1989/90 und 2020 folgende Parallelen

a. Die Unsicherheit als Prinzip des politischen Entscheidens ist das Muster (Korte et al. 2021). Es existiert beim Spitzenpersonal eine Erwartungssicherheit gegenüber dem Nicht-Erwartbaren. Mit der Überraschung zu rechnen, zeichnet Verunsicherungsfähigkeiten bei den Akteuren aus. Die Muster des Entscheidens sind ähnlich, wenn man sich die Krisen-Konstellationen 89/90 und 2020 näher ansieht. Politische Entscheidungen fallen unter extremen Bedingungen von Unsicherheit, Nicht-Wissen und Komplexität. Die Geschwindigkeiten der Entscheidungszumutungen sind rasant und turbulent. Im Modus des tastenden Lernens bildet die jeweilige Regierungsformation professionell Resilienz aus.

b. Glaubenssätze der Politik bleiben unter den Bedingungen des Hochgeschwindigkeitsregierens auf der Strecke. Die über Jahrzehnte als notwendige Bedingung einer Wiedervereinigung geradezu mantrahafte Formulierung: ,nur im Rahmen eines Friedensvertrags“ - blieb folgenlos. Heute könnte man anfügen: offenbar ist 
auch die Globalisierung umkehrbar, wie der weltweite Lockdown im Tagesrhythmus zeigte. Die Globalisierung ist weder robust noch irreversibel.

c. Spezifische Entscheidungsjahre prägen immer die Stunde der Exekutive. Die Legislative ist eher nachgelagert beteiligt und formiert sich mit Parteiendifferenz für Mobilisierungsvorhaben: schnelle oder verlangsamte Einheit; schnelle oder verlangsamte Öffnungen und Ausstiege aus dem Lockdown.

d. Resilienz erwächst vor allem aus der Kraft des Dezentralen, dem Föderalismus und dem Aufbau der Gebietskörperschaften. Resilienz führt idealweise ein System dazu, sich zu transformieren. Heute in Richtung des Vorsorgestaates, damals in Richtung der staatlichen Einheit.

e. Freiheit, konkret Reisefreiheit, war ein zentraler Katalysator der friedlichen Revolution. Die Ostdeutschen erlebten, wie sich die Grammatik der Freiheit ausbuchstabierte. Optionen und neue sicherheitsgetriebene Restriktionen hielten sich zunächst die Waage. Freiheit kam als Beschleunigungsschock daher (Böick 2020). Die Westdeutschen haben erst 2020 erfahren, was es bedeutet, Grundrechte befristet entzogen zu bekommen. Freiheit ging unter den Bedingungen von Entschleunigung dramatisch verloren. Viele spürten erstmals in der Konsequenz des Runterfahrens, wie wertvoll es ist, über das eigene Leben selbst bestimmen zu können. Dialektisch wuchs in der Alltagserfahrung mit der Corona-Politik die Wertschätzung der Freiheit. Gemeinwohlausprägungen (Korte 2019) unter Corona-Solidarität bekamen eine andersartige Ausrichtung. Das sind wichtige Erfahrungen und Diskussionen, die eine Post-Corona-Zeit begleiten, um demokratische Resilienz zu stärken.

Mit Entscheidungszumutungen müssen die Spitzenakteure in der Politik weiterhin umgehen. Bruchlinien des Politikmanagements sieht man besonders deutlich in Entscheidungsjahren.

Funding Open Access funding provided by Projekt DEAL.

Open Access Dieser Artikel wird unter der Creative Commons Namensnennung 4.0 International Lizenz veröffentlicht, welche die Nutzung, Vervielfältigung, Bearbeitung, Verbreitung und Wiedergabe in jeglichem Medium und Format erlaubt, sofern Sie den/die ursprünglichen Autor(en) und die Quelle ordnungsgemäß nennen, einen Link zur Creative Commons Lizenz beifügen und angeben, ob Änderungen vorgenommen wurden.

Die in diesem Artikel enthaltenen Bilder und sonstiges Drittmaterial unterliegen ebenfalls der genannten Creative Commons Lizenz, sofern sich aus der Abbildungslegende nichts anderes ergibt. Sofern das betreffende Material nicht unter der genannten Creative Commons Lizenz steht und die betreffende Handlung nicht nach gesetzlichen Vorschriften erlaubt ist, ist für die oben aufgeführten Weiterverwendungen des Materials die Einwilligung des jeweiligen Rechteinhabers einzuholen.

Weitere Details zur Lizenz entnehmen Sie bitte der Lizenzinformation auf http://creativecommons.org/ licenses/by/4.0/deed.de.

\section{Literatur}

Böick, Marcus. 2020. Die umstrittene Treuhandanstalt. Wirtschaftsumbrüche im Ausnahmezustand gestalten? https://regierungsforschung.de/die-umstrittene-treuhandanstalt/. Zugegriffen: 20.05.2020 
Florack, Martin, Karl-Rudolf Korte, und Julia Schwanholz (Hrsg.). 2021. Coronakratie. Zur Resilienz demokratischen Regierens. Frankfurt a.M., New York: Campus.

Korte, Karl-Rudolf. 1994. Die Chance genutzt? Die Politik zur Einheit Deutschlands. Frankfurt a.M., New York: Campus.

Korte, Karl-Rudolf. 1998. Deutschlandpolitik in Helmut Kohls Kanzlerschaft, Regierungsstil und Entscheidungen 1982-1989. Stuttgart: DVA.

Korte, Karl-Rudolf. 2019. Gesichter der Macht. Über die Gestaltungspotenziale der Bundespräsidenten. Frankfurt a.M., New York: Campus.

Korte, Karl-Rudolf. 2020. Corona-Politik und Politikmanagement. In Deutschland nach Corona, Hrsg. Stefan Iskan. Stuttgart: Kohlhammer.

Korte, Karl-Rudolf, Gert Scobel, und Taylan Yildiz. 2021. Heuristiken des politischen Entscheidens. Zwischen Komplexität und Kunstfertigkeit. Frankfurt a.M.: Suhrkamp. i.E.

Seibel, Wolfgang. 2017. Verwaltung verstehen. Frankfurt a.M.: Suhrkamp.

Weidenfeld, Werner, und Karl-Rudolf Korte. 1991. Die Deutschen. Profil einer Nation. Stuttgart: KlettCotta.

Weidenfeld, Werner, und Karl-Rudolf Korte (Hrsg.). 1999. Handbuch zur deutschen Einheit - 1949-19891999, 4. Aufl. Frankfurt a.M., New York: Campus. 